\title{
Optimization of a high efficiency free electron laser amplifier
}

\author{
E. A. Schneidmiller and M. V. Yurkov" \\ Deutsches Elektronen-Synchrotron (DESY), Notkestrasse 85, D-22607 Hamburg, Germany
}

(Received 4 September 2014; published 9 March 2015)

\begin{abstract}
The free electron laser (FEL) amplifier is implemented in $\mathrm{x}$-ray FEL facilities to generate short wavelength radiation. The problem of an efficiency increase of an FEL amplifier is now of great practical importance. The technique of undulator tapering in the postsaturation regime is used at the existing x-ray FELs LCLS, SACLA and FERMI, and is planned for use at FLASH, European XFEL, Swiss FEL, and PAL XFEL. There are also discussions on the future of high peak and average power FELs for scientific and industrial applications. In this paper we perform a detailed analysis of the tapering strategies for high power seeded FEL amplifiers. Analysis of the radiation properties from the modulated electron beam and application of similarity techniques allows us to derive the universal law of the undulator tapering.
\end{abstract}

DOI: 10.1103/PhysRevSTAB.18.030705

PACS numbers: 41.60.Cr

\section{INTRODUCTION}

Effective energy exchange between the electron beam moving in an undulator and electromagnetic wave happens when the resonance condition takes place. In this case electromagnetic wave advances electron beam by one radiation wavelength while electron beam passes one undulator period. When the amplification process enters the nonlinear stage, the energy losses by electrons become pronounced which leads to the violation of the resonance condition and to the saturation of the amplification process. Application of the undulator tapering [1] allows a further increase of the conversion efficiency. An idea is to adjust undulator parameters (field or period) according to the electron energy loss such that the resonance condition is preserved.

It is generally accepted that in the framework of the onedimensional theory an optimum law of the undulator tapering should be quadratic [2-9]. A similar physical situation occurs in the FEL amplifier with a waveguide [2]. In this case radiation is confined within the waveguide. Parameters of FEL amplifiers operating in the infrared, visible, and $\mathrm{x}$-ray wavelength ranges are such that these devices are described in the framework of three-dimensional theory with an "open" electron beam, i.e., physical case of diffraction in a free space. In this case the diffraction of radiation is an essential physical effect influencing optimization of the tapering process. Discussions and studies on the optimum law of the undulator tapering in the threedimensional case have been in progress for more than 20 years. Our previous studies were mainly driven by

*mikhail.yurkov@desy.de

Published by the American Physical Society under the terms of the Creative Commons Attribution 3.0 License. Further distribution of this work must maintain attribution to the author(s) and the published article's title, journal citation, and DOI. occasional calculations of perspective FEL systems for high power scientific (for instance, FEL based $\gamma \gamma$ collider) and industrial applications (for instance, for isotope separation, and lithography [10-12]). Their parameter range corresponded to the limit of a thin electron beam (small value of the diffraction parameter). In this case linear undulator tapering works well from almost the very beginning [6]. A comprehensive study devoted to the global optimization of a tapered FEL amplifier with open electron beam has been presented in [4]. It has been shown that: (i) the tapering law should be linear for the case of thin electron beam, (ii) optimum tapering at the initial stage should follow quadratic dependence, and (iii) tapering should start approximately two field gain lengths before saturation. A new wave of interest in the undulator tapering came with the development of x-ray free electron lasers [13-20]. Undulator tapering has been successfully demonstrated at long wavelength FEL amplifiers [2,21], and is routinely used at x-ray FEL facilities LCLS and SACLA [16,17]. Practical calculations of specific systems yielded in several empirical laws using different polynomial dependencies (see [22,23] and references therein).

In this paper we perform a comprehensive analysis of the problem of the undulator tapering in the presence of diffraction effects. We found that the key element for understanding the physics of the undulator tapering is given by the model of modulated electron beam. Then we perform global analysis of the parameter space of a seeded FEL amplifier and derive the universal law of the undulator tapering.

\section{BASIC RELATIONS}

We consider an axisymmetric model of the electron beam. It is assumed that the transverse distribution function of the electron beam is Gaussian, so rms transverse size of matched beam is $\sigma=\sqrt{\epsilon \beta}$, where $\epsilon$ is rms beam emittance and $\beta$ is focusing beta function. An important feature of the parameter space of short wavelength FELs is that the space 
charge field does not influence significantly the amplification process, and in the framework of the three-dimensional theory the operation of the FEL amplifier is described by the following parameters: the diffraction parameter $B$, the energy spread parameter $\hat{\Lambda}_{T}^{2}$, the betatron motion parameter $\hat{k}_{\beta}$ and detuning parameter $\hat{C}[9,24]$ :

$$
\begin{aligned}
B & =2 \Gamma \sigma^{2} \omega / c, & \hat{C} & =C / \Gamma, \\
\hat{k}_{\beta} & =1 /(\beta \Gamma), & \hat{\Lambda}_{T}^{2} & =\left(\sigma_{E} / \mathcal{E}\right)^{2} / \rho^{2},
\end{aligned}
$$

where $\Gamma=\left[I \omega^{2} \theta_{s}^{2} A_{\mathrm{JJ}}^{2} /\left(I_{A} c^{2} \gamma_{z}^{2} \gamma\right)\right]^{1 / 2}$ is the gain parameter, $\rho=c \gamma_{z}^{2} \Gamma / \omega$ is the efficiency parameter, and $C=2 \pi / \lambda_{w}-$ $\omega /\left(2 c \gamma_{z}^{2}\right)$ is the detuning of the electron with the nominal energy $\mathcal{E}_{0}$. Note that the efficiency parameter $\rho$ entering equations of three-dimensional theory relates to the onedimensional parameter $\rho_{1 D}$ as $\rho_{1 D}=\rho / B^{1 / 3}[9,25]$. The following notations are used here: $I$ is the beam current, $\omega=2 \pi c / \lambda$ is the frequency of the electromagnetic wave, $\theta_{s}=K / \gamma, K$ is the rms undulator parameter, $\gamma_{z}^{-2}=$ $\gamma^{-2}+\theta_{s}^{2}, \quad k_{w}=2 \pi / \lambda_{w}$ is the undulator wave number, $I_{A}=17 \mathrm{kA}$ is the Alfven current, $A_{J J}=1$ for helical undulator and $A_{J J}=J_{0}\left[K^{2} / 2\left(1+K^{2}\right)\right]-J_{1}\left[K^{2} / 2\left(1+K^{2}\right)\right]$ for planar undulator. $J_{0}$ and $J_{1}$ are the Bessel functions of the first kind. The energy spread is assumed to be Gaussian with rms deviation $\sigma_{E}$.

In the following we consider the case of negligibly small values of the betatron motion parameter $\hat{k}_{\beta}$ and the energy spread parameter $\hat{\Lambda}_{T}^{2}$ (i.e., the case of "cold" electron beam). Under these assumptions the operation of the FEL amplifier is described by the diffraction parameter $B$ and the detuning parameter $\hat{C}$.

Equations describing the motion of the particles in the ponderomotive potential well of the electromagnetic wave and the undulator become simple when written down in the normalized form (see, e.g., [9]):

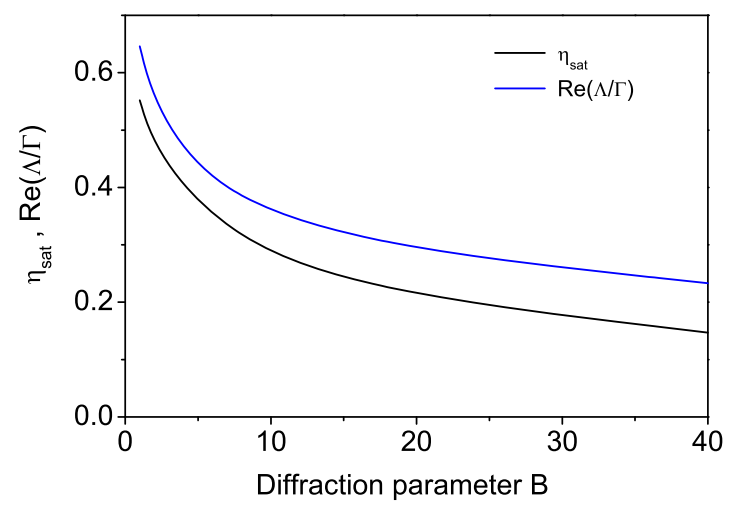

$$
\frac{d \Psi}{d \hat{z}}=\hat{C}+\hat{P}, \quad \frac{d \hat{P}}{d \hat{z}}=U \cos \left(\phi_{U}+\Psi\right),
$$

where $\hat{P}=\left(E-E_{0}\right) /\left(\rho E_{0}\right), \hat{z}=\Gamma z$, and $U$ and $\phi_{U}$ are the amplitude and the phase of the effective potential. Deviation of the electron energy is small in the exponential stage of amplification, $\hat{P} \ll 1$, and the process of the beam bunching in phase $\Psi$ lasts for a long distance, $\hat{z} \gg 1$. The situation changes drastically when the amplification process enters the nonlinear stage and deviation of the electron energy $\hat{P}$ approaches to the unity. The phase change on a scale of $\Delta \hat{z} \simeq 1$ becomes fast, particles start to slip fast in phase $\Psi$ which leads to the reduction of the electron beam modulation, and the growth of the radiation power is saturated.

Field gain length $\operatorname{Re} \Lambda / \Gamma$ and reduced saturation efficiency $\hat{\eta}_{\text {sat }}=W_{\text {sat }} /\left(\rho W_{\text {beam }}\right)$ of the FEL amplifier tuned to exact resonance $\hat{C}=0$ are the functions of the diffraction parameter $B$ and are plotted in Fig. 1 . Here $W_{\text {beam }}=E_{0} I / e$ is the electron beam power. These quantities scale asymptotically as $B^{-1 / 3}$ [9] which gives us a hint for derivation of one more general dependence. Indeed, we normalize the radiation power to the saturation power, and undulator length to the field gain length. Then we find that the radiation power before saturation exhibits similar behavior for all values of the diffraction parameter $B>1$ (see the right plot in Fig. 1).

Undulator tapering [1], i.e., adjustment of the detuning according to the energy loss of electrons, $\hat{C}(\hat{z})=-\hat{P}(\hat{z})$, allows to keep synchronism of trapped electrons with electromagnetic wave. Energy losses of trapped electrons grow proportionally to the detuning. Energy of the particles which are not trapped in the regime of coherent deceleration remains approximately the same as that at the moment when particles leave the stability region. Finally, two fractions of electrons are formed which are well separated in energy, and the average energy loss is $\langle\hat{P}(\hat{z})\rangle \simeq\left\langle\hat{P}_{\text {trap }}(\hat{z})\right\rangle N_{\text {trap }} / N_{\text {tot }}$.

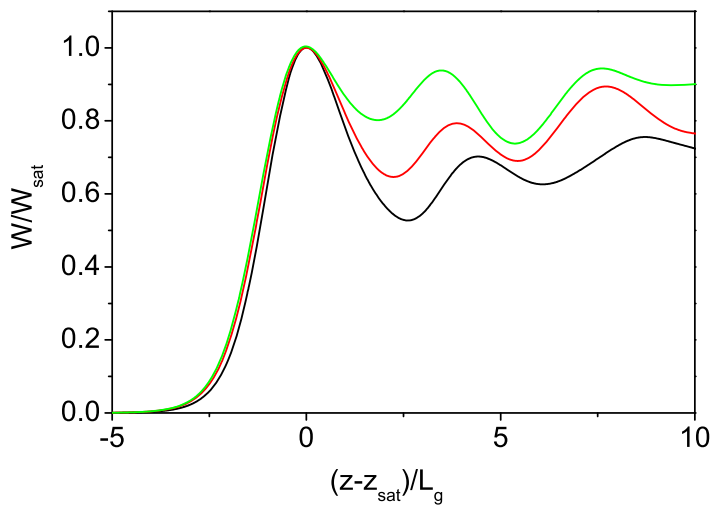

FIG. 1. Operation of the FEL amplifier with untapered undulator. Left plot: FEL field gain $\operatorname{Re} \Lambda / \Gamma$ (black curve) and FEL efficiency at the saturation $\eta_{\text {sat }}=W_{\text {sat }} /\left(\rho W_{\text {beam }}\right)$ (blue curve) versus the diffraction parameter $B$. Right plot: Evolution of the radiation power along the undulator. Black, red, green curves correspond to the value of the diffraction parameter $B=1,10$, and 40 . 
Thus, for large values of $|\langle\hat{P}(\hat{z})\rangle| \gg 1$, the ratio of $|\langle\hat{P}(\hat{z})\rangle| /$ $\hat{C}(\hat{z})=\hat{\eta} / \hat{C}(\hat{z})$ approaches asymptotically the value of the trapping factor of the particles captured in the regime of coherent deceleration $k_{\text {trap }}=N_{\text {trap }} / N_{\text {tot }}$.

\section{A. Radiation of modulated electron beam}

During the amplification process the electron beam is modulated periodically at the resonance wavelength. This modulation grows exponentially in the high gain linear regime, reaching a value about the unity near the saturation point. Application of undulator tapering allows to preserve beam bunching at a long distance. Thus, it is useful to remember the properties of the radiation of the modulated electron beam. Electron beam current $I(z, t)=I_{0}[1+$ $\left.a_{\text {in }} \cos \omega\left(z / v_{z}-t\right)\right]$ is modulated with amplitude $a_{\text {in }}$. Radiation power of the modulated beam is given by [26]

$$
\begin{gathered}
W=\frac{2 \pi^{2} I_{0}^{2} a_{\mathrm{in}}^{2} \sigma^{2}}{c \lambda \lambda_{u}} \frac{K^{2} A_{J J}^{2}}{1+K^{2}} f(\tilde{z}) \tilde{z}, \\
f(\tilde{z})=\arctan (\tilde{z} / 2)+\tilde{z}^{-1} \ln \left(\frac{4}{\tilde{z}^{2}+4}\right) .
\end{gathered}
$$

In the right-hand side of expression (3) we explicitly isolated $z$ dependence of the radiation power with function $f(\tilde{z})$ of argument $\tilde{z}=1 / N$, where $N=k \sigma^{2} / z$ is Fresnel number, and $k=2 \pi / \lambda$ is wave number. A plot of the function $f(\tilde{z})$ is shown in Fig. 2. Asymptotes of the function $f(\tilde{z})$ are

$$
\begin{array}{lll}
f(\tilde{z}) \rightarrow \pi / 2 & \text { for } \tilde{z} \gg 1 & (N \ll 1), \\
f(\tilde{z})=\tilde{z} / 4 & \text { for } \tilde{z} \ll 1 & (N \gg 1)
\end{array}
$$

for thin and wide electron beam asymptote, respectively. Expression (3) is a crucial element for understanding the optimum law of the undulator tapering. Indeed, in the deep tapering regime some fraction of the particles is trapped

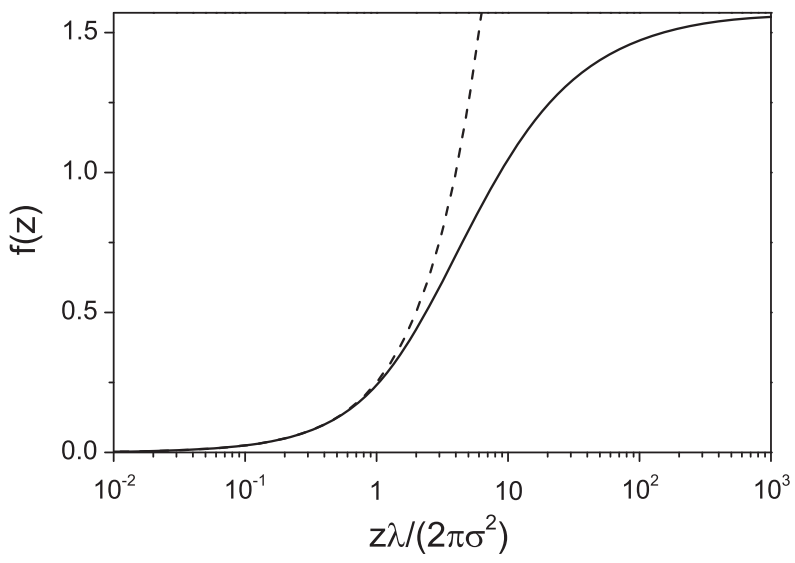

FIG. 2. Function $f(z)$ entering Eq. (3). The dashed line shows the asymptote (4) for small values of $z, f(\tilde{z})=\tilde{z} / 4$. in the regime of coherent deceleration. Thus, the beam modulation is fixed, and the radiation power $W$ should follow the expression (3), and the detuning (undulator tapering) should follow the energy loss by particles, $C \propto W$. One can easily find that both asymptotes (of wide and thin electron beam) discussed in the introductory section are well described by this expression. The asymptote of a wide electron beam corresponds to large values of Fresnel number $N$, and it follows from (3) that the radiation power scales quadratically with the undulator length, $W \propto z^{2}$. The asymptote of a thin electron beam corresponds to small values of the Fresnel number $N$, and the radiation power grows linearly with the undulator length, $W \propto z$. Undulator tapering should adjust detuning according to the energy loss by electrons, and we find that the tapering law should be quadratic for the case of wide electron beam, $C \propto W \propto z^{2}$, and linear for the case of thin electron beam, $C \propto W \propto z$.

The asymptote of the wide electron beam works reasonably well for the values of the Fresnel number $N \gtrsim 1$. The asymptote of the thin electron beam converges pretty slowly, and reasonable accuracy is achieved for very small values of the Fresnel number, $N \lesssim 0.01$. To get the feeling about practical numbers, let us consider two working points of LCLS x-ray FEL operating at the radiation wavelength of 0.15 and $1.5 \mathrm{~nm}[16,27]$. The transverse size of the electron beam is about $25 \mu \mathrm{m}$ in both cases. The wide beam asymptote is applicable up to $z \simeq z_{\mathrm{wb}} \simeq 26 \mathrm{~m}$ for wavelength $0.15 \mathrm{~nm}$, and $z \simeq z_{\mathrm{wb}} \simeq 2.6 \mathrm{~m}$ for operation at $1.5 \mathrm{~nm}$ wavelength. Here we see general feature illustrating shortening with the radiation wavelength of the applicability region of the wide beam asymptote. The thin beam asymptote becomes applicable at LCLS for $z \gtrsim 2500 \mathrm{~m}$ (for wavelength $0.15 \mathrm{~nm}$ ) and $260 \mathrm{~m}$ (for wavelength $1.5 \mathrm{~nm}$ ). Note, that for both practical examples the limit of thin electron beam is achieved only for very long undulator, and exact formula (3) should be used for calculation of the radiation power for undulator length $z>z_{\mathrm{wb}}$.

\section{GLOBAL OPTIMIZATION OF THE UNDULATOR TAPERING}

Now we turn to the problem of finding a general law of the optimum undulator tapering. First, we solve this problem using the approach of straightforward global optimization with three-dimensional, time-dependent FEL simulation code FAST [28]. The target of the optimization is to find the maximum of the output power at the undulator length after ten field gain lengths. We divide the undulator into many pieces and change detuning of all pieces independently. We apply adiabatic (smooth) tapering, i.e., we prevent jumps of the detuning on the boundary of the sections. The number of sections is controlled to be large enough to provide the result which is independent on the number of sections. Then we choose the tapering law $C(B, z)$ corresponding to the maximum 


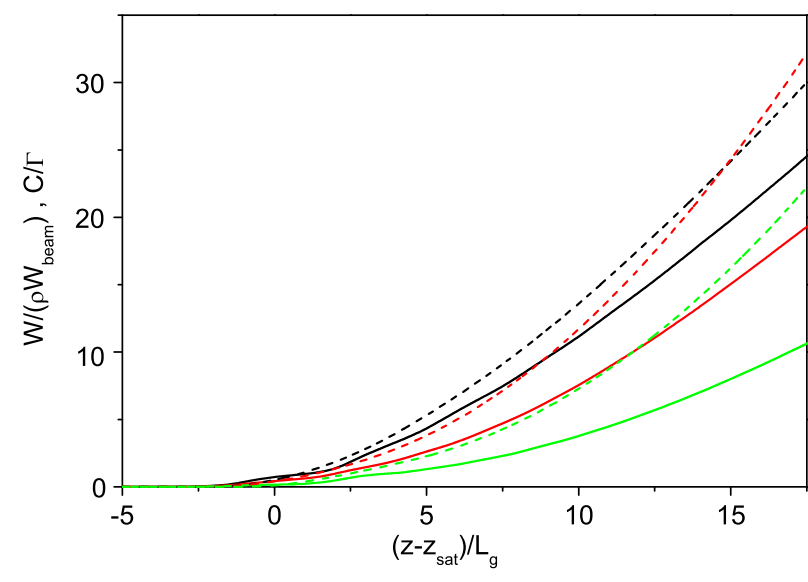

FIG. 3. Evolution along the undulator of the reduced radiation power $\hat{\eta}=W /\left(\rho W_{\text {beam }}\right)$ (solid curves) and of the detuning parameter $\hat{C}=C / \Gamma$ (dashed curves) for the FEL amplifier with global optimization of the undulator tapering. Color codes: black, red, green curves correspond to the value of diffraction parameter $B=1,10$, and 40 .

power at the exit of the whole undulator. This global optimization procedure has been performed in the practically important range of diffraction parameters from $B=1$ to $B=40$. Results of this global optimization are summarized in Fig. 3. Solid curves show the profiles of the optimized radiation power, and dashed curves show the profiles of the optimum detuning. It has been shown in the previous section that the ratio of the normalized radiation power to the normalized detuning gives us the value of the trapping efficiency of the electrons in the regime of coherent deceleration, $K_{\text {trap }}=\hat{\eta} / \hat{C}$. The trapping efficiency is the function of the diffraction parameter $B$ and is plotted in Fig. 4. We find that the optimum trapping factor approaches values of 0.8 for $B=1$, and falls down to 0.45 for $B=40$. It is worth noticing that for $B \gtrsim 5$ it scales roughly as $B^{-1 / 3}$, similar to other FEL characteristics like field gain length and saturation efficiency.

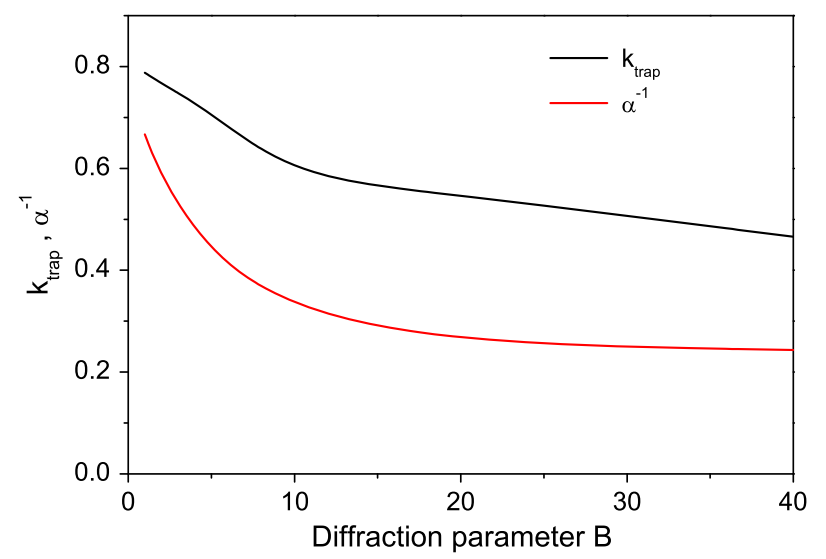

FIG. 4. The trapping efficiency $K_{\text {trap }}$ for the globally optimized undulator (black curve) and the fitting coefficient $\alpha_{\text {tap }}^{-1}$ of the global optimization entering Eq. (5) (red curve).

\section{UNIVERSAL TAPERING LAW}

It follows from the global optimization that in the whole parameter range the undulator tapering starts from the value of $\Delta z \simeq 2 L_{g}$ before saturation. This result is in good qualitative agreement with intuitive analysis of the evolution of the radiation power in the beginning of the nonlinear regime (see Fig. 1). The losses of the electron energy follow identical parametric dependence on the gain $L_{g}$ for all values of the diffraction parameter $B$. Visible losses of the electron energy start to occur approximately about two field gain lengths before saturation. The next observations come from the analysis of the beam modulation. The first observation is that the beam modulation at the initial stage of the nonlinear regime follows similar behavior for all diffraction parameters (see Fig. 5). This gives a hint that initial capture of the particles is performed in a similar way in the whole parameter range. The second observation is that the beam modulation after trapping of the electrons in the coherent deceleration process remains approximately constant along the undulator. This gives us the main hint which we discussed in the previous section. That is, excluding the transition stage of the trapping process, we deal with the radiation of the modulated electron beam (3). The main essence of our study is to apply parametrical dependence like (3) to fit an optimum detuning pattern in Fig. 3 such that the condition of the optimum tapering is preserved:

$$
\hat{C}=\alpha_{\text {tap }}\left(\hat{z}-\hat{z}_{0}\right)\left[\arctan \left(\frac{1}{2 N}\right)+N \ln \left(\frac{4 N^{2}}{4 N^{2}+1}\right)\right],
$$

with Fresnel number $N$ fitted by $N=\beta_{\text {tap }} /\left(\hat{z}-\hat{z}_{0}\right)$. Thus, we try to fit optimum detuning with three parameters: $z_{0}$, $\alpha_{\text {tap }}$ and $\beta_{\text {tap }}$. Here undulator length is normalized to the gain parameter, $\hat{z}=\Gamma z$. One parameter of this fit, start of

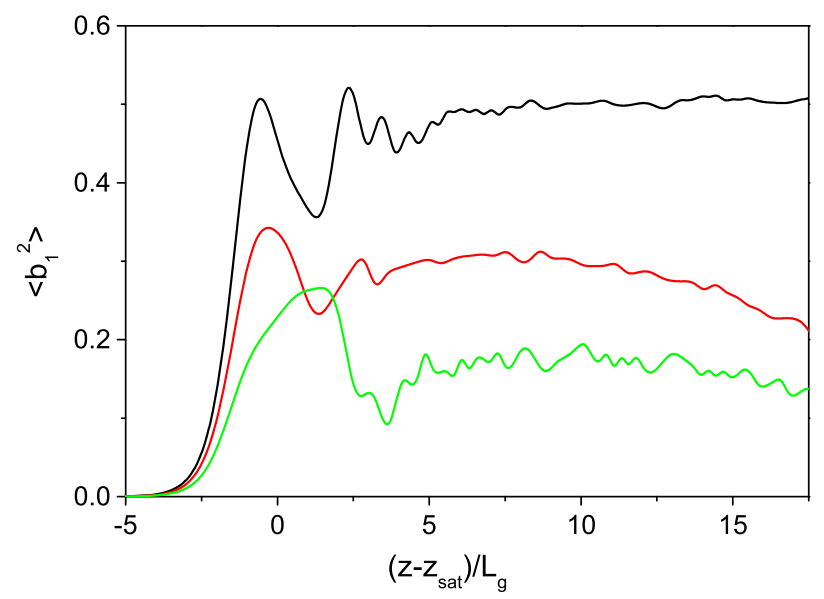

FIG. 5. Evolution along the undulator of the squared value of the bunching factor for the FEL amplifier with global undulator tapering. Color codes: black, red, green curves correspond to the value of the diffraction parameter $B=1,10$, and 40 . 


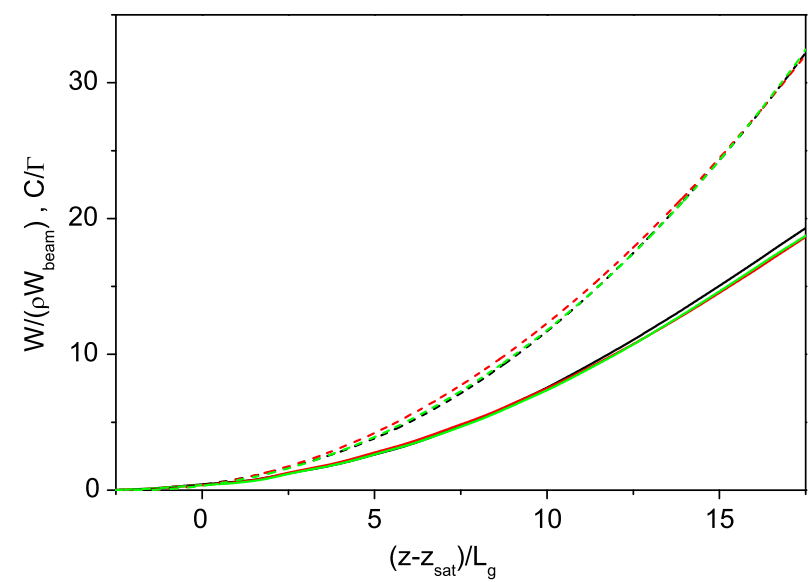

FIG. 6. Evolution along the undulator of the reduced radiation power $\hat{\eta}=W /\left(\rho W_{\text {beam }}\right)$ (solid curves) and of the detuning parameter $\hat{C}=C / \Gamma$ (dashed curves). Color codes: black-FEL with global optimization of undulator tapering; red-fit with the formula (5); green - fit with the rational function (6). Here the value of the diffraction parameter is $B=10$.

the undulator tapering $z_{0}$ is fixed by the global optimization procedure, $z_{0}=z_{\mathrm{sat}}-2 L_{g}$. Another parameter of the problem, $\beta_{\text {tap }}$, is rather well approximated with the linear dependency on diffraction parameter, $\beta_{\text {tap }}=8.5 \times B$. The remaining parameter, $\alpha_{\text {tap }}$, is plotted in Fig. 4 . It is a slow varying function of the diffraction parameter $B$, and scales approximately to $B^{1 / 3}$ as all other important FEL parameters discussed above. Thus, application of similarity techniques gives us an elegant way for the general parametrical fit. The accuracy of this fit is pretty good giving the results for optimum detuning which are close to the global optimum. We illustrate with Fig. 6 tapering law (5) for the specific value of the diffraction parameter $B=10$. Curves in black color are the normalized power and the detuning parameter derived from the global optimization. The red dashed curve is detuning $\hat{C}$ given by (5) with $\alpha_{\text {tap }}=3.6$ (see Fig. 4), and $\beta_{\text {tap }}=85$ (according to relation $\beta_{\text {tap }}=$ $8.5 \times B)$. The solid curve in red color is the normalized FEL efficiency simulated with the detuning pattern (5). We see a good agreement of the fit with the global optimization. The same situation occurs in the whole range of the traced values of the diffraction parameter $B$. Such a good agreement is not surprising since fitting is based on very clean parametric dependencies, and numerical simulations just provided relevant numerical factors.

\section{A. Rational fit}

Analysis of the expression (5) shows that it has quadratic dependence in $z$ for small values of $z$ (limit of the wide electron beam), and linear dependence in $z$ for large values of $z$ (limit of the thin electron beam). It is natural to try a fit with a rational function which satisfies both asymptotes. The simplest rational fit is

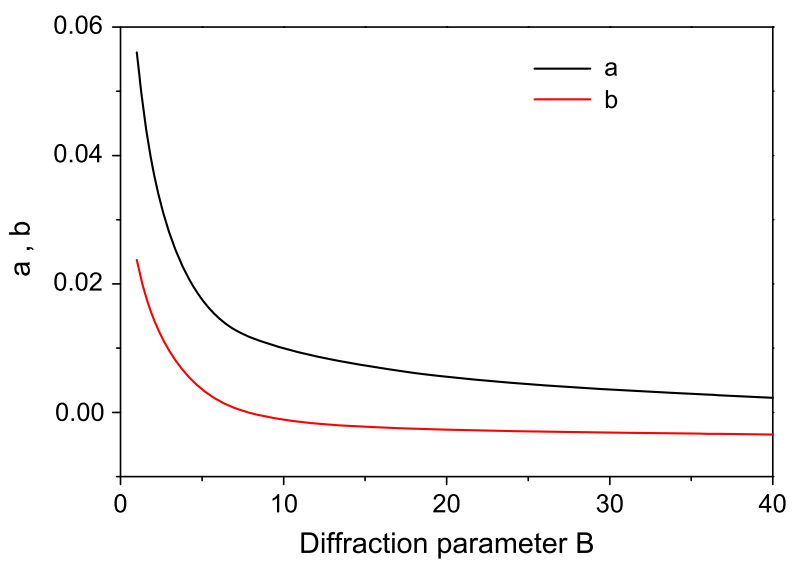

FIG. 7. Coefficients $a$ (black line) and $b$ (red line) of the rational fit of the tapering law (6).

$$
\hat{C}=\frac{a\left(\hat{z}-\hat{z}_{0}\right)^{2}}{1+b\left(\hat{z}-\hat{z}_{0}\right)} .
$$

The coefficients $a$ and $b$ are the functions of the diffraction parameter $B$, and are plotted in Fig. 7. The start of the undulator tapering is set to the value $z_{0}=z_{\text {sat }}-2 L_{g}$ suggested by the global optimization procedure. Analysis of the plots presented in Fig. 6 shows that the fit of the tapering law with the rational function also works well.

\section{B. Trapping process}

We finish our paper with the illustration of the trapping process. The trapping efficiency $K_{\text {trap }}=\hat{P} / \hat{C}$ falls down with the diffraction parameter $B$ (see Fig. 4 ). This is a natural consequence of the diffraction effects discussed in earlier publications (see, e.g., Ref. [9], Chapter 4). Indeed, FEL radiation is not a plane wave. The transverse distribution of the radiation field (FEL radiation mode $[9,29]$ ) depends on the value of the diffraction parameter $B$, and the

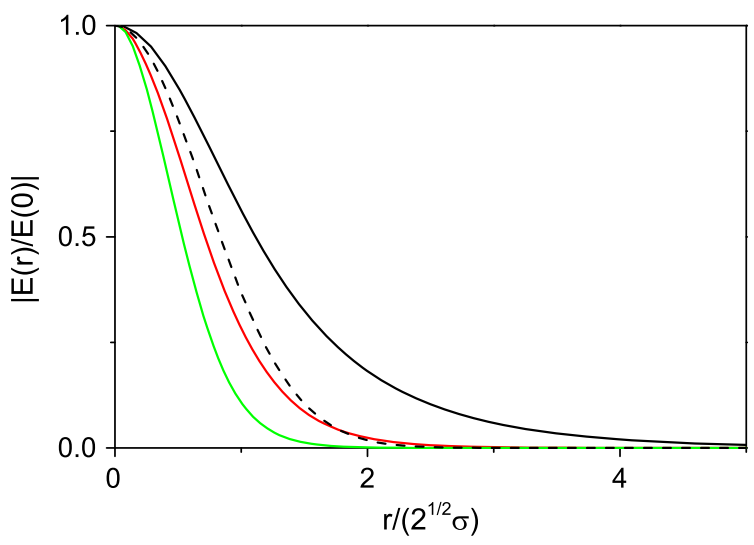

FIG. 8. Field amplitude of the FEL radiation mode in the high gain linear regime. Black, red, and green colors correspond to the value of the diffraction parameter $B=1,10$, and 40 , respectively. The dashed line shows the profile of the beam current density $\exp \left[-r^{2} /\left(2 \sigma^{2}\right)\right]$. 

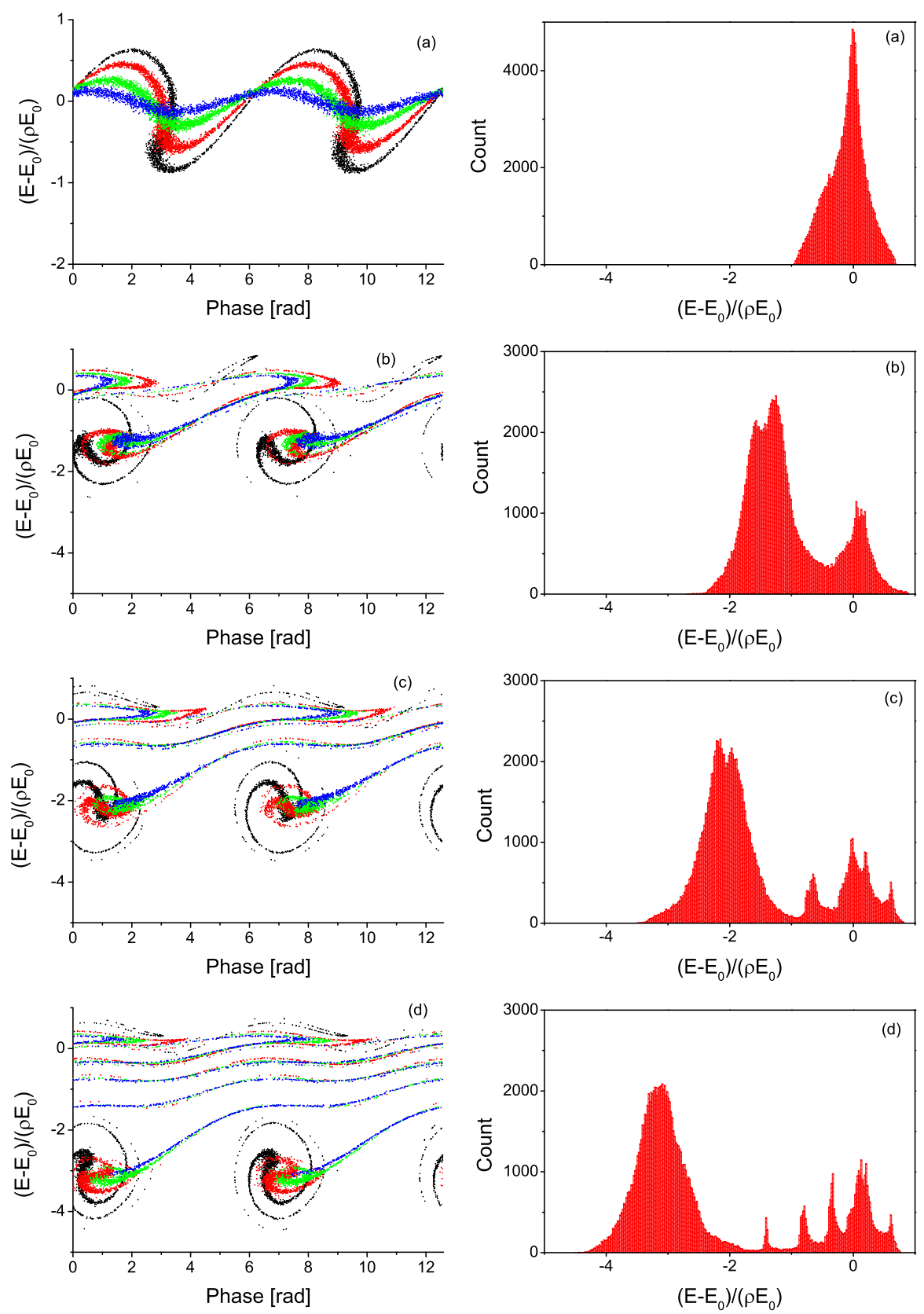

FIG. 9. Phase space distribution of electrons (left column) and population of electrons in energy (right column) at different stages of the trapping process. Color codes correspond to different locations of the particles in the beam (black-core of the beam; blue-edge of the beam). Here diffraction parameter is $B=10$. Plots labeled by (a), (b), (c), and (d) correspond to $\left(z-z_{\text {sat }}\right) / L_{g}=-1.2,2.5,3.9$ and 5.3, respectively (see Fig. 6).

field gradient (or, amplitude of ponderomotive well) across the electron beam is more pronounced for larger values of the diffraction parameter $B$ (see Fig. 8). In the latter case we obtain a situation when electrons located in the core of the electron beam are already fully bunched while electrons at the edge of the beam are not bunched yet [see phase space plot (a) in Fig. 9]. As a result, the number of electrons with similar positions on the energy-phase plane falls down with the growth of the diffraction parameter, as well as the trapping efficiency in the regime of coherent deceleration.
The trapping process is illustrated with the phase space plots presented in Fig. 9 for the value of the diffraction parameter $B=10$. Different color codes (black to blue) correspond to different locations of the particles across the beam (from the beam core to the edge). We see that the particles in the core of the beam (black points) are trapped most effectively. Nearly all particles located at the edge of the electron beam (blue points) leave the stability region very soon. The trapping process lasts for a several field gain lengths when the trapped particles become isolated in the 
trapped energy band for which the undulator tapering is optimized further. For the specific value of the diffraction parameter $B=10$ the trapping process is not finished even at three field gain lengths after saturation, and nontrapped particles continue to populate the low energy tail of the energy distribution (see the right column of Fig. 9). There was an interesting experimental observation at LCLS that energy distribution of nontrapped particles is not uniform, but represent a kind of energy bands [30-32]. Graphs presented in Fig. 9 give a hint on the origin of energy bands which are formed by nontrapped particles. This is the consequence of nonlinear dynamics of electrons leaving the region of stability. Note that a similar effect can be seen in the early one-dimensional studies $[7,8]$.

\section{DISCUSSION}

In this paper we derived the general law (5) for the optimum undulator tapering in the presence of diffraction effects. The case of the cold electron has been considered. This allowed us to isolate diffraction effects in the most clear form. It has been found that the optimum tapering law is the function of the only diffraction parameter $B$. Fit of the tapering law with the rational function (6) requires fulfillment of two asymptotes of the tapering law: quadratic at the initial stage (wide beam asymptote), and linear for very long tapering section (thin beam asymptote). It is essentially simple, and can be very convenient for optimization of practical systems. The tapering law is described by simple analytical expressions with two fitting coefficients. Extension of this approach to a more complicated model (including energy spread and emittance) is pretty much straightforward and will result in corrections to the fitting coefficients without changing the general law given by (5).

\section{ACKNOWLEDGMENTS}

We are grateful to William Fawley for many useful discussions. We are grateful to Vadim Banine and Vivek Bakshi; contacts with them and other members of the industrial community stimulated our interest to the development of high power FEL systems. We thank Christopher Behrens for attracting our attention to deeper analysis of the trapping process (energy bands) and for useful discussions.

[1] N. M. Kroll, P. L. Morton, and M. N. Rosenbluth, IEEE J. Quantum Electron. 17, 1436 (1981).

[2] T. J. Orzechowski, B. R. Anderson, J. C. Clark, W. M. Fawley, A. C. Paul, D. Prosnitz, E. T. Scharlemann, S. M. Yarema, D. B. Hopkins, A. M. Sessler, and J. S. Wurtele, Phys. Rev. Lett. 57, 2172 (1986).

[3] R. A. Jong, E. T. Scharlemann, and W. M. Fawley, Nucl. Instrum. Methods Phys. Res., Sect. A 272, 99 (1988).

[4] W. M. Fawley, Nucl. Instrum. Methods Phys. Res., Sect. A 375, 550 (1996).
[5] W. M. Fawley, Z. Huang, K. J. Kim, and N. Vinokurov, Nucl. Instrum. Methods Phys. Res., Sect. A 483, 537 (2002).

[6] E. L. Saldin, E. A. Schneidmiller, and M. V. Yurkov, Opt. Commun. 95, 141 (1993).

[7] E. T. Scharlemann, Laser Handbook, Volume 6, Free Electron Lasers, edited by W. B. Colson, C. Peilegrini, and A. Renieri (North-Holland, Amsterdam, 1991), p. 291.

[8] E. L. Saldin, E. A. Schneidmiller, and M. V. Yurkov, Phys. Rep. 260, 187 (1995).

[9] E. L. Saldin, E. A. Schneidmiller, and M. V. Yurkov, The Physics of Free Electron Lasers (Springer-Verlag, Berlin, 1999).

[10] E. L. Saldin, V. P. Sarantsev, E. A. Schneidmiller, and M. V. Yurkov, Nucl. Instrum. Methods Phys. Res., Sect. A 339, 583 (1994).

[11] C. Pagani, E. L. Saldin, E. A. Schneidmiller, and M. V. Yurkov, Nucl. Instrum. Methods Phys. Res., Sect. A 455, 733 (2000).

[12] E. A. Schneidmiller, V. F. Vogel, H. Weise, and M. V. Yurkov, J. Micro/Nanolithogrphy, MEMS, MOEMS 11, 021122 (2012).

[13] W. Ackermann et al., Nat. Photonics 1, 336 (2007).

[14] M. Vogt, B. Faatz, J. Feldhaus, K. Honkavaara, S. Schreiber, and R. Treusch, in Proceedings of the IPAC 2014 Conference, Dresden, Germany, 2014, TUOCA02, http://accelconf.web.cern.ch/AccelConf/IPAC2014/papers/ tuoca02.pdf.

[15] E. Allaria et al., Nat. Photonics 6, 699 (2012).

[16] P. Emma et al., Nat. Photonics 4, 641 (2010).

[17] T. Ishikawa et al., Nat. Photonics 6, 540 (2012).

[18] XFEL: The European X-Ray Free-Electron Laser, edited by Massimo Altarelli et al., DESY Technical Design Report No. 2006-097, DESY, Hamburg, 2006, see also http://xfel.desy.de/technical_information/tdr/tdr/.

[19] Swiss FEL Conceptual Design Report, edited by R. Ganter, PSI Bericht No. 10-04, 2012.

[20] H. S. Kang, K. W. Kim, and I. S. Ko, in Proceedings of the IPAC 2014 Conference, THPRO019, http://accelconf .web.cern.ch/AccelConf/IPAC2014/papers/thpro019.pdf.

[21] X. J. Wang, H. P. Freund, D. Harder, W. H. Miner, Jr., J. B. Murphy, H. Qian, Y. Shen, and X. Yang, Phys. Rev. Lett. 103, 154801 (2009).

[22] Y. Jiao, J. Wu, Y. Cai, A. W. Chao, W. M. Fawley, J. Frisch, Z. Huang, H.-D. Nuhn, C. Pellegrini, and S. Reiche, Phys. Rev. ST Accel. Beams 15, 050704 (2012).

[23] G. Geloni, V. Kocharyan, and E. Saldin, DESY Report No. 11-049, 2011.

[24] E. L. Saldin, E. A. Schneidmiller, and M. V. Yurkov, Nucl. Instrum. Methods Phys. Res., Sect. A 475, 86 (2001).

[25] R. Bonifacio, C. Pellegrini, and L. M. Narducci, Opt. Commun. 50, 373 (1984).

[26] E. L. Saldin, E. A. Schneidmiller, and M. V. Yurkov, Nucl. Instrum. Methods Phys. Res., Sect. A 539, 499 (2005).

[27] J. Galayda, in Proceedings of the International Particle Accelerator Conference, Kyoto, Japan (ICR, Kyoto, 2010), MOYAMH01, http://accelconf.web.cern.ch/ AccelConf/IPAC10/papers/moyamh01.pdf. 
[28] E. L. Saldin, E. A. Schneidmiller, and M. V. Yurkov, Nucl. Instrum. Methods Phys. Res., Sect. A 429, 233 (1999).

[29] E. T. Scharlemann, A. M. Sessler, and J. S. Wurtele, Nucl. Instrum. Methods Phys. Res., Sect. A 239, 29 (1985).

[30] C. Behrens, The Workshop on Advanced X-Ray FEL Development, 2014, Hamburg (European XFEL GmbH, Hamburg, 2014), http://www.xfel.eu/sites/site_xfel-gmbh/ content/e63594/e63599/e228764/e228774/e233398/Trex XTCAVandFELtrappingobservations_Behrens_DIST OK_eng.pdf.
[31] P. Krejcik, in Proceedings of the IPAC 2014 Conference, Dresden, Germany, 2014, WEOBB03, http://accelconf .web.cern.ch/AccelConf/IPAC2014/talks/weobb03_talk .pdf.

[32] Y. Ding, F.-J. Decker, V. A. Dolgashev, J. Frisch, Z. Huang, P. Krejcik, H. Loos, A. Lutman, A. Marinelli, T. J. Maxwell, D. Ratner, J. Turner, J. Wang, M.-H. Wang, J. Welch, and J. Wu, SLAC National Accelerator Laboratory Report No. SLAC-PUB-16105, 2014. 